\title{
AN INDEPENDENCE RESULT IN SEVERAL COMPLEX VARIABLES
}

\author{
LÁSZLÓ LEMPERT AND LEE A. RUBEL
}

(Communicated by Andreas R. Blass)

\begin{abstract}
The assertion that there exists a complete set of biholomorphic invariants for simply connected domains in $\mathbb{C}^{2}$ is proved to be independent of the axioms (ZFC) of set theory, give or take the Continuum Hypothesis.
\end{abstract}

\section{INTRODUCTION}

In this paper, we study the assertion, "There exists a complete system of biholomorphic invariants for simply connected domains of holomorphy in $\mathbb{C}^{2}$ " and show that it is independent of the axioms (ZFC) of set theory, give or take the Continuum Hypothesis $(\mathrm{CH})$. That is, there exists a model of $(\mathrm{ZFC} \pm \mathrm{CH})$ in which the assertion is true, but there exists another model in which it is false. In [BHR], the corresponding assertion for multiply connected domains in $\mathbb{C}^{1}$ with "conformal equivalence" replacing "biholomorphic equivalence" was studied, with the same (in-)conclusion. In $\S 1$ of the present paper, we refer heavily to [BHR] rather than repeat large sections of it.

We remind the reader that a region $\Omega$ in $\mathbb{C}^{n}$ is a domain of holomorphy if there is a holomorphic function on $\Omega$ that has no holomorphic extension to a proper open superset of $\Omega$. See [KRA, Theorem 3.4.2, p. 125] for some equivalent versions of this statement, including that $\Omega$ be pseudoconvex. The pseudoconvex domains form a very natural class of study. We do not know whether our results remain true for strictly pseudoconvex simply connected domains. In [LEM], the first author constructed a complete set of biholomorphic invariants, within any model of ZFC, for marked (or pointed) strictly convex and smoothly bounded regions in $\mathbb{C}^{n}$, for any $n$. We do not know whether there exists a complete system of biholomorphic invariants for marked pseudoconvex or strictly pseudoconvex regions in $\mathbb{C}^{n}$, even for $n=1$. In any event, our construction of the class of domains $D_{\alpha}$, which, in one model of ZFC, are not classified up to biholomorphic equivalence by any system of invariants, is inconclusive as soon as these particular domains are marked. These domains

Received by the editors April 16, 1990.

1980 Mathematics Subject Classification (1985 Revision). Primary 03C25, 32D05.

The research of both authors was partially supported by grants from the National Science Foundation. 
$D_{\alpha}$ suffer from no particular pathology; they are all diffeomorphic to the unit ball

$$
B=\left\{\left(z_{1}, z_{2}\right):\left|z_{1}\right|^{2}+\left|z_{2}\right|^{2}<1\right\} .
$$

The main contribution of the present paper is the construction of a class of simply connected domains of holomorphy $D_{\alpha}$, one for each subset $\alpha$ of $\mathbb{Z}$, such that $D_{\alpha}$ is biholomorphically equivalent to $D_{\beta}$ if and only if there is a transformation $T$ of $\mathbb{Z}$ onto $\mathbb{Z}$ of the form $T(z)= \pm z+b$, some $b=$ $b(T) \in \mathbb{Z}$, such that $T(\alpha)=\beta$. This construction is carried out in $\S \S 2-6$. In $\S 1$, quoting heavily from [BHR], we show how this construction can be used to prove independence, the main fact from logic being that, in a suitable model of set theory, there is no complete set of invariants for sets of integers under the above group of transformations.

\section{LOGICAL CONSIDERATIONS}

We quote from [BHR]:

The importance of the Zermelo-Fraenkel axioms with the Axiom of Choice (the $Z F C$ axiom system) is that they seem to capture all of our present intuitions about the mathematical universe of sets. Moreover, experience bears out this impression very strongly, in that all existing mathematical arguments can easily be formalized and proved from the $Z F C$ axioms. Thus to prove that a mathematical assertion $S$ is consistent with the $Z F C$ axioms is to show that one could not prove the negation of $S$ using any of the currently available methods of mathematics.

As stated in the Introduction, we will rely heavily on [BHR, §6] where all the details are to be found. We shall start with a countable transitive model $(M, \varepsilon)$ of the $Z F C$ axioms. For $S$, a set of integers generic over $M$, let $(M[S], \varepsilon)$ denote the smallest transitive model of $Z F C$ which contains $M \cup\{S\}$. For the nonlogician, it will probably suffice to believe that there is a model $M^{\prime}=$ $M[S]$ for which the following lemma holds. (Such a model exists with the continuum hypothesis and with its negation.) This lemma is the logical heart of our argument.

Lemma 1.1. Let $S \subseteq \mathbb{Z}$ be a generic set over $M$, and let $S_{0}, S_{1} \subseteq \mathbb{Z}$ be defined by $S_{0}=\{n \in \mathbb{Z}: 2 n \in S\}$ and $S_{1}=\{n \in \mathbb{Z}: 2 n+1 \in S\}$. Then

(1) There is no mapping of the form $T(z)=a z+b$ which maps $S_{0}$ onto $S_{1}$.

(2) If $F\left(x, y_{1}, \ldots, y_{n}\right)$ is a formula in the language of set theory such that for each $m \in \mathbb{Z}$ the formula

$$
\forall z \subseteq \mathbb{Z}\left(F\left(z, y_{1}, \ldots, y_{n}\right) \Leftrightarrow F\left(z+m, y_{1}, \ldots, y_{n}\right)\right)
$$


is provable from the $Z F C$ axioms, then the equivalence

$$
F\left(S_{0}, a_{1}, \ldots, a_{n}\right) \Leftrightarrow F\left(S_{1}, a_{1}, \ldots, a_{n}\right)
$$

is true in $(M[S], \varepsilon)$ for each $a_{1}, \ldots, a_{n} \in M$.

The proof of Lemma 1.1, which is [BHR, Lemma 6.1], can be found there.

We will call a formula $F\left(x, y_{1}, \ldots, y_{n}\right)$ in the language of set theory biholomorphically invariant if the following assertion can be proved from the $Z F C$ axioms:

If $\Omega$ and $\Omega^{\prime}$ are biholomorphically equivalent domains and if $y_{1}, \ldots, y_{n}$ are arbitrary parameters, then $F\left(\Omega, y_{1}, \ldots, y_{n}\right) \Leftrightarrow$ $F\left(\Omega^{\prime}, y_{1}, \ldots, y_{n}\right)$.

We remind the reader that a biholomorphic equivalence between $\Omega$ and $\Omega^{\prime}$ is a one-one holomorphic mapping of $\Omega$ onto $\Omega^{\prime}$ whose inverse is also holomorphic.

Theorem 1.2. Let $S \subseteq \mathbb{Z}$ be a generic set over $M$ and let $S_{0}, S_{1} \subseteq \mathbb{Z}$ be defined by $S_{0}=\{n \in \mathbb{Z}: 2 n \in S\}$ and $S_{1}=\{n \in \mathbb{Z}: 2 n+1 \in S\}$. For each $i=0,1$, let $\Omega$ be the domain $D_{S_{i}}$ constructed in the later sections of the present paper. Then:

(1) $\Omega_{0}$ and $\Omega_{1}$ are not biholomorphically equivalent in the model $(M[S], \varepsilon)$.

(2) For any biholomorphically invariant formula $F\left(x, y_{1}, \ldots, y_{n}\right)$ and any parameters $a_{1}, \ldots, a_{n}$ from $M$, the equivalence

$$
F\left(\Omega_{0}, a_{1}, \ldots, a_{n}\right) \Leftrightarrow F\left(\Omega_{1}, a_{1}, \ldots, a_{n}\right)
$$

holds in the model $(M[S], \varepsilon)$.

The proof of Theorem 1.2 is the same as the proof of [BHR, Theorem 6.2] but uses Theorem 6.1 of the present paper instead of the conformal mapping argument at the beginning of the proof of [BHR, Theorem 6.2].

Definition 1.3. The group $G$ is the group of transformations $T: \mathbb{Z} \rightarrow \mathbb{Z}$ of the form $T(z)= \pm z+b$, for $b \in \mathbb{Z}$.

This group is called the shift-flip group for obvious reasons.

Next, we quote from [BHR, p. 162ff], replacing "conformal" by "biholomorphic" throughout.

Let Seq denote the class of all well-ordered sequences of complex numbers. That is, if $\varphi \in$ Seq, then the domain of $\varphi$ is an ordinal number $\rho$ and $\varphi$ is a mapping from $\rho$ into $\mathbb{C}$. (Recall that $\rho=\{\eta: \eta<\rho\}$.) For the purposes of this paper, a system of invariants is an operator $\Phi$ which assigns to each simply connected domain of holomorphy $\Omega \subseteq \mathbb{C}^{n}$ a sequence $\varphi(=\Phi(\Omega))$ of complex numbers. That is, $\Phi$ is a mapping from the set of all simply connected domains of holomorphy into Seq. We require, of course, that $\Phi(\Omega)$ should be an invariant of $\Omega$ : whenever $\Omega$ and $\Omega^{\prime}$ are biholomorphically equivalent, then we should have $\Phi(\Omega)=\Phi\left(\Omega^{\prime}\right)$. Also we require that the 
definition of $\Phi$ should be definite and explicit. We take this to mean that there exists a formula $R(x, y)$ in the language of set theory such that, for any simply connected domain of holomorphy $\Omega$ and any $\varphi \in$ Seq,

$$
\Phi(\Omega)=\varphi \Leftrightarrow R(\Omega, \varphi) .
$$

A system of invariants is complete if each simply connected domain holomorphy $\Omega$ is determined up to biholomorphic equivalence by $\Phi(\Omega)$. That is, whenever $\Phi(\Omega)=\Phi\left(\Omega^{\prime}\right)$, then $\Omega^{\prime}$ must be biholomorphically equivalent to $\Omega$. This formulation of the vague concept of invariants for regions is very broad apparently, broader than any invariants which have been considered in classical complex analysis.

Theorem 1.4. Let $S$ be any generic subset of $\mathbb{Z}$ and consider the model $(M[S], \varepsilon)$ of the ZFC axioms. Construct (simply connected) domains of holomorphy $\Omega_{0}$, $\Omega_{1}$ as in Theorem 1.2. Then, for any system of biholomorphic invariants $\Phi$ defined in $M[S]$ by a formula of set theory, we have

$$
\Phi\left(\Omega_{0}\right)=\Phi\left(\Omega_{1}\right) .
$$

On the other hand, $\Omega_{0}$ is not biholomorphically equivalent to $\Omega_{1}$ in $M[S]$.

The proof is the same as the proof of [BHR, Theorem 6.7].

We now prove that it is consistent with $Z F C \pm C H$ that there does exist a complete system of biholomorphic invariants for (arbitrary) regions in $\mathbb{C}^{n}$, for any $n$, say $n=2$. In particular, we show that this happens if there exists a wellordering of $\mathscr{P}(\mathbb{N})$ which is definable using second-order logic over the structure $(\mathbb{N},+, \cdot) .($ Here, $\mathbb{N}$ is the set of all natural numbers, and $\mathscr{P}(\mathbb{N})=\{A: A \subseteq \mathbb{N}\}$ is the power set of $\mathbb{N}$.) It is known that there is a model of the $Z F C$ axioms (give or take $C H$ ) which contains such a well-ordering of $\mathscr{P}(\mathbb{N})$.

Theorem 1.5. It is consistent with the ZFC axioms for set theory (and with the Continuum Hypothesis or its negation) to assume that there exists a complete system of biholomorphic invariants for all domains in $\mathbb{C}^{n}$, for any $n=1,2,3, \ldots$

The proof of this result is the same as parts of the proof of [BHR, Theorem 7.1] except that, for dimensions $n>1$, the coding of a domain into Seq requires more parameters. The idea of the proof, for any domain $\Omega$, is to let $\Omega_{0}$ be the unique domain biholomorphically equivalent to $\Omega$ for which $\mathscr{R}\left(\Omega_{0}\right)$ is least possible under the assumed well-ordering. Here, $\mathscr{R}(\Omega)$ is a simple process for coding any region $\Omega$ into a sequence of rational numbers.

With the logic part of the proof attended to, we now turn to the analytic part-the construction of the domains $D_{\alpha}$ and the proofs of their desired properties.

\section{IMBEDDING THE GROUP $G$ INTO Aut $B$}

Let $r_{1}=(e-1) /(e+1)$, and define a biholomorphic automorphism $T$ of the unit ball

$$
B=\left\{\left(z_{1}, z_{2}\right) \in \mathbb{C}^{2}:\left|z_{1}\right|^{2}+\left|z_{2}\right|^{2}-1<0\right\}
$$


by

$$
T\left(z_{1}, z_{2}\right)=\left(\frac{z_{1}+r_{1}}{1+r_{1} z_{1}}, \sqrt{1-r_{1}^{2}} \frac{z_{2}}{1+r_{1} z_{1}}\right)
$$

(see [KRA]). $T$ maps $(0,0)$ to $\left(r_{1}, 0\right)$; more generally, it preserves the $z_{1}$ plane and the $\operatorname{Re} z_{1}$ axis. Iterating $T$, we find

$$
T^{\nu}\left(z_{1}, z_{2}\right)=\left(\frac{z_{1}+r_{\nu}}{1+r_{\nu} z_{1}}, \sqrt{1-r_{\nu}^{2}} \frac{z_{2}}{1+r_{\nu} z_{1}}\right) \quad(\nu=0, \pm 1, \ldots),
$$

where $r_{\nu}=\left(e^{\nu}-1\right) /\left(e^{\nu}+1\right)$. The points $r_{\nu}$ sit equidistantly on the real axis if distance is measured in the Poincare metric of the unit disc in $\mathbb{C}$. Consecutive $r_{\nu}$ 's are at Poincaré distance 1.

Introduce furthermore the automorphism

$$
S\left(z_{1}, z_{2}\right)=\left(-z_{1}, z_{2}\right) \text {. }
$$

Proposition 2.1. The group generated by $S$ and $T$ is isomorphic to $G$.

Proof. Any holomorphic function or map defined on $B$ is uniquely determined by its restriction to the manifold

$$
M=\left\{\left(z_{1}, z_{2}\right) \in B: z_{1} \in \mathbb{R}, z_{2} \in \mathbb{R}\right\} .
$$

Hence it suffices to prove that the restrictions of $S$ and $T$ to $M$ generate a group isomorphic to $G$. Now this latter statement becomes clear if $M$ is regarded as the Cayley-Klein model of the hyperbolic plane, for in this model $T$ is translation by one unit along some line and $S$ is reflection into a line perpendicular to the first line.

\section{Construction of the Domains $D_{\alpha}$}

Let $\alpha \subset \mathbb{Z}$. We shall associate with this set a subdomain $D_{\alpha}$ of $B$ in such a way that $D_{\alpha}$ and $D_{\beta}$ are biholomorphic if and only if $\alpha$ and $\beta$ are equivalent under $G$. Moreover, $D_{\alpha}$ will be topologically a ball, and a domain of holomorphy.

Pick first a function $\varphi \in C^{\infty}(\mathbb{C})$ such that

(1) $\varphi(\zeta)=\varphi(|\zeta|) \geq 0$;

(2) $\operatorname{supp} \varphi=\{\zeta: d(0, \zeta) \leq 1 / 8\}, d$ denoting the Poincare distance in the unit disc in $\mathbb{C}$; and

(3) the partials of order $\leq 2$ of $\varphi$ are everywhere $<\varepsilon$, where $\varepsilon$ is some fixed small positive number. For example, $\varepsilon=10^{-6}$ will certainly do.

Define for $j=1,2$ the domains

$$
B_{j}=\left\{z \in \mathbb{C}^{2}:\left|z_{1}\right|^{2}+\left|z_{2}\right|^{2}+j \varphi\left(z_{1}\right)-1<0\right\},
$$

Then $B_{2} \subset B_{1} \subset B$, and both $B_{1}, B_{2}$ are strictly convex. Now, for $\alpha \subset \mathbb{Z}$ put

$$
D_{\alpha}=\bigcap_{\nu \in \alpha} T^{\nu}\left(B_{1}\right) \cap \bigcap_{\nu \in \mathbb{Z} \backslash \alpha} T^{\nu}\left(B_{2}\right) .
$$


The intersection of $B_{1}$ resp. $B_{2}$ with planes $z_{1}=$ const $(\mid$ const $\mid<1)$ are discs whose centers lie on the plane $z_{2}=0$. Since $T^{\nu}$ respects this property of discs, it follows that the same holds for $D_{\alpha}$. In particular, $D_{\alpha}$ is diffeomorphic to the product of two discs, hence also to $B$ itself.

Next we claim that $D_{\alpha}$ is a domain of holomorphy. Indeed, $B_{1}$ and $B_{2}$ are convex, hence domains of holomorphy. It follows that $T^{\nu}\left(B_{j}\right)$ are also domains of holomorphy, hence so is $D_{\alpha}$, an intersection of domains of holomorphy.

We shall now investigate boundary points $w=\left(w_{1}, w_{2}\right) \in \partial D_{\alpha}$ more closely. Clearly $w \in \bar{B}$; hence $\left|w_{1}\right| \leq 1$. Assume first that $\left|w_{1}\right|<1$ and there is no $\nu \in \mathbb{Z}$ such that

$$
d\left(w_{1}, r_{\nu}\right) \leq 1 / 8 .
$$

Then $w \in \partial B$; moreover, in a neighborhood of $w, \partial B$ and $\partial D_{\alpha}$ agree.

If $\left|w_{1}\right|<1$ and there is a $\nu$ such that (3.1) holds, then this $\nu$ is uniquely determined, $w \in T^{\nu}\left(\partial B_{1}\right)$ or $w \in T^{\nu}\left(\partial B_{2}\right)$, and in a neighborhood of $w$, $\partial D_{\alpha}$ agrees with $T^{\nu}\left(\partial B_{1}\right)$ or $T^{\nu}\left(\partial B_{2}\right)$. However, in no neighborhood of $w$ will $\partial D_{\alpha}$ and $\partial B$ agree.

If $\left|w_{1}\right|=1, w_{1} \neq \pm 1$, then again, in a small neighborhood of $w, \partial D_{\alpha}$ and $\partial B$ agree. Finally, if $w_{1}= \pm 1-$ i.e., $w=( \pm 1,0)$ - then in no neighborhood of $w$ will $\partial D_{\alpha}$ and $\partial B$ agree, although, of course, $w \in \partial B$. From this analysis it follows that $\partial D_{\alpha}$ is a $C^{\infty}$ hypersurface except at the two points $( \pm 1,0)$.

For the purposes of this paper we shall introduce the notion of spherical resp. nonspherical boundary points of $D_{\alpha}$. A point $w \in \partial D_{\alpha}$ will be called spherical if there is a neighborhood $V$ of $w$ such that

$$
V \cap \partial D_{\alpha}=V \cap \partial B
$$

otherwise, $w$ will be called nonspherical. Thus $w=\left(w_{1}, w_{2}\right) \in \partial D_{\alpha}$ is spherical if and only if $\left|w_{1}\right|=1$ but $w_{1} \neq \pm 1$, or $\left|w_{1}\right|<1$ but for no $\nu \in \mathbb{Z}$ is (3.1) satisfied.

\section{Extremal Discs}

We shall study biholomorphisms between the domains $D_{\alpha}$ using the device of extremal discs. Let $D \subset \mathbb{C}^{2}$ be a bounded domain, $p \in D$, and $v \in \mathbb{C}^{2}$ a vector. Consider the family $\mathscr{F}$ of all holomorphic mappings of the unit disc $U \subset \mathbb{C}$

$$
f: U \rightarrow D
$$

such that $f(0)=p$ and $f^{\prime}(0)=\lambda v$, with some $\lambda=\lambda_{f}>0$. Call an $f \in \mathscr{F}$ an extremal mapping if $\lambda_{f} \geq \lambda_{g}$ for every $g \in \mathscr{F}$. In this case $f(U)$ resp. $\overline{f(U)}$ will be called an extremal disc resp. closed extremal disc. Extremal mappings can be used to study biholomorphic maps because of the following simple fact: Let $F$ be a biholomorphic mapping between two domains $D$ and $\widetilde{D}, p \in D$, $v \in \mathbb{C}^{2}, \tilde{p}=F(p)$, and $\tilde{v}=F^{\prime}(p) v$. If $f: U \rightarrow D$ is an extremal mapping 
corresponding to $p, v$, then $\tilde{f}=F \circ f: U \rightarrow \widetilde{D}$ is an extremal mapping corresponding to $\tilde{p}, \tilde{v}$.

Extremal mappings corresponding to some point $p \in D$ and vector $v \in \mathbb{C}^{2}$ may or may not exist; if they exist, they are not necessarily unique. However, in the case when $D$ is the ball $B$, they do exist and are easy to characterize.

Proposition 4.1. There is a unique extremal map $f: U \rightarrow B$ associated with any pair $p \in B, v \in \mathbb{C}^{2}$. The corresponding extremal disc is the intersection of $B$ with the complex line

$$
L_{p, v}=\{p+\zeta v: \zeta \in \mathbb{C}\} .
$$

The proof is well known: one first settles the case when $p=0$ (using Schwarz's lemma), and then reduces the general case to this special one by applying an automorphism of $B$ and invoking the holomorphic invariance of extremality.

It is not that easy to describe all extremal discs in the domains $D_{\alpha}$ we are primarily interested in here. However, a description of some of them is a straightforward consequence of Proposition 4.1:

Proposition 4.2. Suppose for some complex line $L=L_{p, v}$ as in (4.1), $p \in D_{\alpha}$, $v \in \mathbb{C}^{2}$, we have

$$
D_{\alpha} \cap L=B \cap L \neq \varnothing .
$$

Then $D_{\alpha} \cap L$ is an extremal disc in $D_{\alpha}$, and the unique extremal map $f: U \rightarrow$ $D_{\alpha}$ determined by $p, v$ agrees with the extremal map $U \rightarrow B$ determined by the same data.

Indeed, this proposition follows from the fact that $D_{\alpha} \subset B$.

\section{AUTOMORPHISMS OF $D_{\alpha}$}

The group of biholomorphic automorphisms of $D_{\alpha}$, equipped with the compact-open topology (i.e., the topology of locally uniform convergence) forms a topological group Aut $D_{\alpha}$. Denote the component of the identity by $\operatorname{Aut}_{0} D_{\alpha}$. For example, rotations

$$
R_{\theta}:\left(z_{1}, z_{2}\right) \mapsto\left(z_{1}, e^{i \theta} z_{2}\right) \quad(\theta \in \mathbb{R})
$$

belong to $\operatorname{Aut}_{0} D_{\alpha}$.

Lemma 5.1. $\operatorname{Aut}_{0} D_{\alpha}=\left\{R_{\theta}: \theta \in \mathbb{R}\right\}$.

Proof. Since the rotation group $\left\{R_{\theta}\right\}$ is a closed subgroup of the connected group $\mathrm{Aut}_{0} D_{\alpha}$, in order to prove that they agree it would suffice to show that the rotation subgroup is open; or, equivalently, that id is an interior point of it. In other words, assuming that an automorphism $F: D_{\alpha} \rightarrow D_{\alpha}$ is sufficiently near to id, we have to prove that $F=R_{\theta}$ for some $\theta$.

Let $F \in$ Aut $D_{\alpha}$ be near id. 
Proposition 5.2. $F$ extends to an automorphism of $B$.

Proof. Take a point $w_{1} \in U$ such that $d\left(r_{\nu}, w_{1}\right)>1 / 8$ for every $\nu \in \mathbb{Z}$. Then the disc

$$
\Delta_{w_{1}}=\left\{\left(w_{1}, \zeta\right):|\zeta|<\sqrt{1-\left|w_{1}\right|^{2}}\right\}
$$

is an extremal disc in $B$ and therefore, by Proposition 4.2 , also in $D_{\alpha}$; it is the extremal disc determined by the point $p=\left(w_{1}, 0\right)$ and the vector $v=(0,1)$.

Choose now an $\omega \in U$ and a positive $\varepsilon$ so that the above holds whenever $\left|\omega-w_{1}\right| \leq \varepsilon$. Notice that the closures of the extremal discs $\Delta_{w_{1}}$ all meet $\partial D_{\alpha}$ in spherical points; moreover, the intersections of $\Delta_{w_{1}}$ with $\partial D_{\alpha}$ sweep out an open portion of the set of spherical points.

Suppose now that $F$ is so near to id that the complex lines $L=L_{F(p), F^{\prime}(p) v}$ intersect $\partial D_{\alpha}$ in spherical points whenever $w_{1}$ is such that $\left|\omega-w_{1}\right| \leq \varepsilon$. In this case $D_{\alpha} \cap L_{F(p), F^{\prime}(p) v}=\widetilde{\Delta}_{w_{1}}$ is an extremal disc in $D_{\alpha}$, so that by the invariance of extremal discs we see that

$$
F\left(\Delta_{w_{1}}\right)=\tilde{\Delta}_{w_{1}},
$$

and thus $F$ is a biholomorphism between the domains

$$
\Omega_{1}=\bigcup\left\{\Delta_{w_{1}}:\left|w_{1}-\omega\right|<\varepsilon\right\}
$$

and

$$
\Omega_{2}=\bigcup\left\{\tilde{\Delta}_{w_{1}}:\left|w_{1}-\omega\right|<\varepsilon\right\} .
$$

Recall now the following theorem of Rudin [RUD]:

Theorem 5.3. Suppose $\Omega_{1}, \Omega_{2}$ are subdomains of $B$ such that both $\partial \Omega_{1}$ and $\partial \Omega_{2}$ intersect $\partial B$ and the intersections $\Gamma_{1}, \Gamma_{2}$ have nonempty interior (relative interior in $\partial B$ ). Let $a, b$ be points of $\Gamma_{1}$ resp. $\Gamma_{2}$ which are not limit points of $B \cap \partial \Omega_{1}$ resp. $B \cap \partial \Omega_{2}$, and let $a_{k} \in \Omega_{1}, b_{k} \in \Omega_{2}$ be sequences that converge to a resp. $b$.

If $F$ is a biholomorphic map between $\Omega_{1}$ and $\Omega_{2}$ such that $F\left(a_{k}\right)=b_{k}$, then $F$ extends to a biholomorphic automorphism of $B$.

In our situation, choosing any convergent sequence $a_{k} \in \Delta_{\omega}, a_{k} \rightarrow a \in \partial D_{\alpha}$, and putting $b_{k}=F\left(a_{k}\right) \in \widetilde{\Delta}_{\omega}$, we can apply the above theorem to conclude that $F$ indeed extends to an automorphism of $B$.

We can now return to the proof of Lemma 5.1. From Proposition 5.2 it follows that $F$ holomorphically extends past $\bar{D}_{\alpha}$ and, indeed, past $\bar{B}$. We shall denote the extension by the same letter $F$. Since $F\left(\partial D_{\alpha}\right)=\partial D_{\alpha}$ and $F(\partial B)=\partial B, F$ maps spherical points to spherical points; hence, it smoothly maps the boundary of the spherical set into itself. The boundary of the spherical set consists of the union of the tori

$$
X_{\nu}=\left\{\left(z_{1}, z_{2}\right): d\left(r_{\nu}, z_{1}\right)=1 / 8,\left|z_{2}\right|=\sqrt{1-\left|z_{1}\right|^{2}}\right\},
$$

and the two points $( \pm 1,0)$. 
It follows that $F$ either fixes these two points or exchanges them. In either case $F$ leaves the complex line joining $( \pm 1,0)$ invariant, since any automorphism of $B$ preserves complex lines (see, e.g., [KRA] or [RUD]).

Next, consider the set $X_{0}$. This is a connected component of the boundary of the spherical set, so $F\left(X_{0}\right)=X_{\nu}$ for some $\nu$. However, since $F$ is near the identity, $\nu$ will have to be $0: F\left(X_{0}\right)=X_{0}$. One easily checks that this implies that $F$ also preserves the polynomial hull of $X_{0}$ (note that both $F$ and $F^{-1}$ can be uniformly approximated on $B$ by polynomials). The polynomial hull of the torus

$$
X_{0}=\left\{z:\left|z_{1}\right|=q,\left|z_{2}\right|=\sqrt{1-q^{2}}\right\},
$$

where $q=\left(e^{1 / 8}-1\right) /\left(e^{1 / 8}+1\right)$ is the bidisc

$$
\widehat{X}_{0}=\left\{z:\left|z_{1}\right| \leq q,\left|z_{2}\right| \leq \sqrt{1-q^{2}}\right\} .
$$

Hence $F$ restricts to an automorphism of the bidisc int $\widehat{X}_{0}$.

Now the automorphisms of a bidisc (near the identity) are known to be of form

$$
F\left(z_{1}, z_{2}\right)=\left(f\left(z_{1}\right), g\left(z_{2}\right)\right),
$$

where $f, g$ are automorphisms of the corresponding disc factors (see [KRA]). Since we already know that the $z_{1}$ axis is preserved by $F, g(0)=0$, whence $g\left(z_{2}\right)=e^{i \theta} z_{2}$. On the other hand, $f$ preserves both discs, $\left|z_{1}\right|<1$ and $\left|z_{1}\right|<$ $q$, whence $f\left(z_{1}\right)=e^{i t} z_{1} . F( \pm 1,0)=( \pm 1,0)$ now implies $f\left(z_{1}\right)= \pm z_{1}$, with the negative sign excluded by the assumption of $F$ being near the identity. In conclusion,

$$
F\left(z_{1}, z_{2}\right)=\left(z_{1}, e^{i \theta} z_{2}\right),
$$

which was to be proved.

\section{Equivalence Between $D_{\alpha}$ AND $D_{\beta}$}

Theorem 6.1. Suppose $D_{\alpha}$ and $D_{\beta}$ are biholomorphically equivalent. Then $\alpha$ and $\beta$ are G-equivalent.

This theorem follows from the following, more precise statement:

Proposition 6.2. Let $H$ be a biholomorphism between $D_{\alpha}$ and $D_{\beta}$. Then $H$ extends to an automorphism of $B$, and is of one of the following two forms:

$$
\begin{gathered}
H=T^{n} \circ R_{\theta}, \\
H=S \circ T^{n} \circ R_{\theta},
\end{gathered}
$$

with $n \in \mathbb{Z}, \theta \in \mathbb{R}$. 
Proof that $H$ is an automorphism of $B$. For some $\theta \in \mathbb{R}$ consider the automorphism $K: D_{\beta} \rightarrow D_{\beta}$ defined by

$$
K=H \circ R_{\theta} \circ H^{-1} .
$$

When $\theta=0, K=i d$, so for any $\theta, K \in$ Aut $_{0} D_{\beta}$; hence, by Lemma $5.1, K$ is some rotation $R_{\sigma}$. Thus

$$
H \circ R_{\theta}=R_{\sigma} \circ H .
$$

If $H=(h, k)$, this means that

$$
\begin{aligned}
& h\left(z_{1}, e^{i \theta} z_{2}\right)=h\left(z_{1}, z_{2}\right), \\
& k\left(z_{1}, e^{i \theta} z_{2}\right)=e^{i \sigma} k\left(z_{1}, z_{2}\right) .
\end{aligned}
$$

Taylor expansion in the $z_{2}$ variable then shows (put, e.g., $\theta=1$ ) that

$$
h\left(z_{1}, z_{2}\right)=\gamma\left(z_{1}\right), \quad k\left(z_{1}, z_{2}\right)=\kappa\left(z_{1}\right) z_{2}^{n},
$$

with some nonnegative integer $n$ and holomorphic functions $\gamma, \kappa$. Since $H$ is biholomorphic, $n$ has to be 1 ; i.e.,

$$
H\left(z_{1}, z_{2}\right)=\left(\gamma\left(z_{1}\right), \kappa\left(z_{1}\right) z_{2}\right) .
$$

Putting $z_{2}=0$, we conclude that $\gamma$ is an automorphism of the unit disc $U$, so that it preserves the Poincaré distance.

The set

$$
\Sigma=\bigcup_{\nu \in \mathbb{Z}}\left\{z_{1} \in U: d\left(r_{\nu}, z_{1}\right) \leq 1 / 8\right\} \subset U
$$

has 0 density (measured in the Poincare metric). Therefore $\Sigma$ and $\gamma^{-1}(\Sigma)$ together cannot cover $U$; choose any $\zeta \in U \backslash\left(\Sigma \cup \gamma^{-1}(\Sigma)\right)$. Then the point $z=\left(\zeta, \sqrt{1-|\zeta|^{2}} e^{i \tau}\right)(\tau \in \mathbb{R})$ is a spherical boundary point of $D_{\alpha}$, and its image

$$
H(z)=\left(\gamma(\zeta), \kappa(\zeta) \sqrt{1-|\zeta|^{2}} e^{i \tau}\right)
$$

is a spherical boundary point of $D_{\beta}$. It follows that an open subset of the spherical points of $\partial D_{\alpha}$ is mapped by the biholomorphism $H$ into the set of spherical points on $\partial D_{\beta}$; hence, by [RUD, Theorem 5.3], $H$ extends to an automorphism of $B$.

Proof that $H$ is of form (6.1) or (6.2). We can argue now as in the proof of Lemma 5.1. Since (the holomorphic extension of) $H$ maps $\partial D_{\alpha}$ to $\partial D_{\beta}$ and $\partial B$ to $\partial B$, it follows that (non)spherical points on $\partial D_{\alpha}$ are mapped to (non)spherical points on $\partial D_{\beta}$. A point $\left(z_{1}, z_{2}\right) \in \partial D_{\alpha}$ (or $\left.\partial D_{\beta}\right)$ is nonspherical if and only if $z_{1} \in \Sigma$. Hence, $\gamma(\Sigma)=\Sigma$.

Now $\Sigma$ is a disjoint union of discs whose (hyperbolic) centers are $r_{\nu}$. It follows that $\gamma$ preserves the set $\left\{r_{\nu}: \nu \in \mathbb{Z}\right\}$. Therefore $\gamma$ is either of form

$$
\gamma(\zeta)=t^{n}(\zeta) \equiv \frac{\zeta+r_{n}}{1+r_{n} \zeta} \quad(n \in \mathbb{Z})
$$


or of form

$$
\gamma(\zeta)=s(\zeta) \equiv-\zeta
$$

or of form

$$
\gamma=s \circ t^{n} \quad(n \in \mathbb{Z}) .
$$

This last case clearly includes the second case.

Assume the first case holds; i.e., $\gamma$ is a hyperbolic translation $t^{n}$. Then $T^{-n} \circ H$ is an automorphism of $B$ which fixes 0 . By Schwarz's lemma, this implies that $T^{-n} \circ H$ is a unitary map (see [RUD]). In fact, $T^{-n} \circ H$ is the identity on the $z_{1}$ axis, so

$$
T^{-n} \circ H\left(z_{1}, z_{2}\right)=\left(z_{1}, e^{i \theta} z_{2}\right)=R_{\theta}\left(z_{1}, z_{2}\right),
$$

with some $\theta \in \mathbb{R}$; i.e., (6.1) holds.

In the same way one can show that (6.5) implies (6.2). This proves Proposition 6.2.

Proof of Theorem 6.1. Suppose $H$ is a biholomorphic equivalence between $D_{\alpha}$ and $D_{\beta}$. Then it is of form (6.1) or (6.2). Assume (6.1). [(6.2) can be treated in the same way.] Since $R_{\theta}$ is an automorphism of $D_{\alpha}$, we have

$$
T^{n}\left(D_{\alpha}\right)=D_{\beta} \text {. }
$$

We claim that a $\nu \in \mathbb{Z}$ belongs to $\alpha$ if and only if $\nu+n \in \beta$; i.e., $\alpha$ and $\beta$ are translates of one another. Indeed, for any $\nu \in \mathbb{Z}$ the two sets

$$
T^{-\nu}\left(D_{\alpha}\right) \cap\left\{z_{1}=0\right\}
$$

and

$$
T^{-\nu-n}\left(D_{\beta}\right) \cap\left\{z_{1}=0\right\}
$$

agree. However, from the definition of $D_{\alpha}, D_{\beta}$ we see that they are $B_{1} \cap\left\{z_{1}=\right.$ $0)\}$ or $B_{2} \cap\left\{z_{1}=0\right\}$ according to whether, in the case of (6.6), $\nu \in \alpha$ or $\nu \in \mathbb{Z} \backslash \alpha$, resp. in the case of (6.7), $\nu+n \in \beta$ or $\nu+n \in \mathbb{Z} \backslash \beta$. This proves our claim, and with it the theorem.

\section{REFERENCES}

[BHR] J. Becker, C. W. Henson, and L. A. Rubel, First order conformal invariants, Ann. of Math. (2) 112 (1980), 123-178.

[KRA] S. G. Krantz, Function theory of several complex variables, Wiley, New York, 1982.

[LEM] L. Lempert, Holomorphic invariants, normal forms and the moduli space of convex domains, Ann. of Math. (2) 128 (1988), 43-78.

[RUD] W. Rudin, Function theory on the unit ball of $\mathbb{C}^{n}$, Springer, New York, 1980.

Department of Mathematics, Purdue University, West Lafayette, Indiana 47907

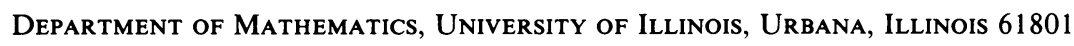

